\title{
Mitochondrial Dysfunction: A Basic Mechanism in Inflammation-Related Non-Communicable Diseases and Therapeutic Opportunities
}

\author{
Anna Hernández-Aguilera, ${ }^{1}$ Anna Rull, ${ }^{1}$ Esther Rodríguez-Gallego, ${ }^{1}$ Marta Riera-Borrull, ${ }^{1}$ \\ Fedra Luciano-Mateo, ${ }^{1}$ Jordi Camps, ${ }^{1}$ Javier A. Menéndez, ${ }^{2}$ and Jorge Joven ${ }^{1}$ \\ ${ }^{1}$ Unitat de Recerca Biomèdica, Hospital Universitari Sant Joan, Institut d'Investigació Sanitària Pere Virgili, \\ Universitat Rovira i Virgili, carrer Sant Llorenç 21, 43201 Reus, Spain \\ ${ }^{2}$ Catalan Institute of Oncology and Girona Biomedical Research Institute, Avda de Francia s/n, 1707 Girona, Spain \\ Correspondence should be addressed to Jorge Joven; jjoven@grupsagessa.com
}

Received 5 December 2012; Revised 1 February 2013; Accepted 1 February 2013

Academic Editor: Fábio Santos Lira

Copyright ( 2013 Anna Hernández-Aguilera et al. This is an open access article distributed under the Creative Commons Attribution License, which permits unrestricted use, distribution, and reproduction in any medium, provided the original work is properly cited.

\begin{abstract}
Obesity is not necessarily a predisposing factor for disease. It is the handling of fat and/or excessive energy intake that encompasses the linkage of inflammation, oxidation, and metabolism to the deleterious effects associated with the continuous excess of food ingestion. The roles of cytokines and insulin resistance in excessive energy intake have been studied extensively. Tobacco use and obesity accompanied by an unhealthy diet and physical inactivity are the main factors that underlie noncommunicable diseases. The implication is that the management of energy or food intake, which is the main role of mitochondria, is involved in the most common diseases. In this study, we highlight the importance of mitochondrial dysfunction in the mutual relationships between causative conditions. Mitochondria are highly dynamic organelles that fuse and divide in response to environmental stimuli, developmental status, and energy requirements. These organelles act to supply the cell with ATP and to synthesise key molecules in the processes of inflammation, oxidation, and metabolism. Therefore, energy sensors and management effectors are determinants in the course and development of diseases. Regulating mitochondrial function may require a multifaceted approach that includes drugs and plant-derived phenolic compounds with antioxidant and anti-inflammatory activities that improve mitochondrial biogenesis and act to modulate the AMPK/mTOR pathway.
\end{abstract}

\section{Background}

The burden of noncommunicable diseases is increasing as such diseases are now responsible for more than three in five deaths worldwide. Atherosclerosis and cancer, in which tobacco use and excessive energy intake are determining factors, are the most frequently occurring of these diseases and are potentially preventable $[1,2]$. Obesity and associated metabolic disturbances, which have been increasing worldwide in recent years, are the main factors that underlie noncommunicable diseases and are the consequences of unhealthy diets and physical inactivity [3]. Approximately $10-20 \%$ of patients with severe obesity, defined as a body mass index $(\mathrm{BMI})>40$, present with no other metabolic complications. These patients are referred to by the oxymoronic designation of "metabolically healthy" obese [47]. Such a designation implies that most obese patients are not "metabolically healthy. " Hence, risk factors for the appearance of noncommunicable diseases have emerged. The reasons for these two phenotypes are unknown; the phenotypes might represent different transitions on a disease timeline, and different levels of either chronic inflammation or insulin resistance are likely contributors. Other contributors include gradual differences in glucose tolerance, inflammatory responses, adipose tissue distribution, patterns of adipokine secretion, and age.

Emerging obesogenic factors are likely to present with significant differences in the elderly, and consequently the 
prevalence of obesity is expected to increase with increasing age. Therefore, it is likely not coincidental that most comorbidity associated with obesity and hence with noncommunicable diseases correlates with aging; the processes may share basic mechanisms, particularly mitochondrial age within an individual [7]. Of note, the prevalence of obesity is lower in people over 70 years of age, an effect attributed to the selective mortality of middle-aged people [8].

Current recommendations to decrease food intake and increase physical exercise do result in metabolic improvements, but such lifestyle changes are rarely sustained, despite strong motivation. However, several communities have undertaken initiatives to prevent noncommunicable diseases, and the lessons learned from the implementation of such initiatives should be examined further [9]. The active manipulation of energy sensors and effectors might be a possible alternative therapeutic procedure. Our aim is to provide a succinct review of the scarce and disseminated data that link mitochondrial dysfunction to the pathogenesis of energy-related complications and to discuss a possible multifaceted therapeutic approach.

\section{Food Availability Links Mitochondrial Dysfunction and the Vicious Cycle of Oxidative Stress and Inflammation}

Mitochondrial defects, systemic inflammation, and oxidative stress are at the root of most noncommunicable diseases such as cancer, atherosclerosis, Parkinson's disease, Alzheimer's disease, other neurodegenerative diseases, heart and lung disturbances, diabetes, obesity, and autoimmune diseases [10-16]. Obesity and obesity-related complications as well as impairment of mitochondrial function, which is required for normal metabolism and health (Figure 1), are universally associated with these conditions. The exact mechanisms that associate mitochondrial dysfunction, obesity, and aging with metabolic syndrome remain a topic of debate [17-22].

Body weight is controlled by molecular messengers that regulate energy status in a limited number of susceptible tissues, including the liver, adipose tissue, skeletal muscles, pancreas, and the hypothalamus [7, 23]. Mouse models of diet-induced obesity have revealed important morphological and molecular differences with respect to humans, particularly those related to the development of fatty liver (NAFLD: nonalcoholic fatty liver disease) or nonalcoholic steatohepatitis (NASH) [24-30] (Figure 2). High expectations for a human therapy after the generation of leptin-deficient animals $(\mathrm{Ob} / \mathrm{Ob})$ were countered by the determination that leptin is not a therapeutic option in humans [28].

Endoplasmic reticulum (ER) and mitochondrial stress, with the consequent oxidative stress, are immediate consequences of attempts to store excess food energy [23, 29]. Under normal weight conditions, adipose tissue-derived adipokines maintain the homeostasis of glucose and lipid metabolism; however, in obese conditions, the dysregulated production of adipokines favours the development of metabolic syndrome and related complications, particularly the accumulation of triglycerides in nonadipose organs that are not designed to store energy [19]. Other adipokines may cause inflammation and oxidative stress [31], but unknown factors are involved because interventions to ameliorate insulin resistance do not lead uniformly to clinical improvement [32]. It is of paramount importance to understand the mechanisms that disrupt ER homeostasis and lead to the activation of the unfolded protein response and mitochondrial defects in metabolic diseases in order to correctly manage noncommunicable diseases [33].

Incidentally, the role of genetics in low-energy expenditure and chronic food intake, although potentially significant, remains poorly understood $[29,30]$. The genetic-selection hypothesis, which attempts to explain the high prevalence of obesity and diabetes in humans, remains controversial, since the recent abandonment of the "thrifty" gene hypothesis [34-38]. As a result, the roles of oxidative stress, inflammation, mitochondrial dysfunction, nutritional status, and metabolism might be reinforced in hypotheses regarding the pathogenesis of noncommunicable diseases (Figures 3 and 4).

Inflammation plays a vital role in host defence. Tissue damage, fibrosis, and losses of function occur under chronic inflammatory conditions. Growing evidence links a lowgrade, chronic inflammatory state to obesity and its coexisting conditions as well as to noncommunicable diseases [1016]. This low-grade inflammatory state is aggravated by the recruitment of inflammatory cells, mainly macrophages, to adipose tissue. Inflammatory cell recruitment is likely due to the combined effects of the complex regulatory network of cells and mediators that are designed to resolve inflammatory responses [7]. Anti-inflammatory drugs have shown to reverse insulin resistance and other related conditions that result from circulating cytokines that cause and maintain insulin resistance $[19,23,39-42]$. Therefore, it is likely that inflammation per se is a causal factor for noncommunicable diseases rather than an associated risk factor.

It is also important to highlight that adipose tissue is comprised of multiple types of cells that have intrinsic and important endocrine functions, particularly those mediated by leptin and adiponectin. Recruited and resident macrophages secrete the majority of inflammatory adipokines, specifically tumour necrosis factor $\alpha$ (TNF $\alpha)$, interleukin-6 (IL-6), and monocyte chemoattractant protein1 (MCP-1), among others. The major roles of TNF $\alpha$ and other inflammatory cytokines in the progression of metabolic complications are likely related to oxidative stress $[43,44]$. In adipose tissue macrophages, increased concentrations of saturated free fatty acids (FFAs) stimulate the synthesis of TNF $\alpha$ directly through the Toll-like receptor 4 (TLR4) or indirectly through cellular accumulation. Both macrophages and adipocytes possess TLR4 receptors that, upon lipiddependent activation, induce NF-KB translocation to the nucleus and the subsequent synthesis of TNF $\alpha$ and IL- 6 $[7,43,44]$. However, recruited macrophages have unique inflammatory properties that are not observed in resident tissue macrophages, and the recruitment of these cells is mainly modulated by MCP-1, the most important molecule of the CC chemokine family [7]. In this setting, the roles and polarisation of adipose tissue macrophages (ATMs) seem established [45]. M1 or "classically activated" ATMs 


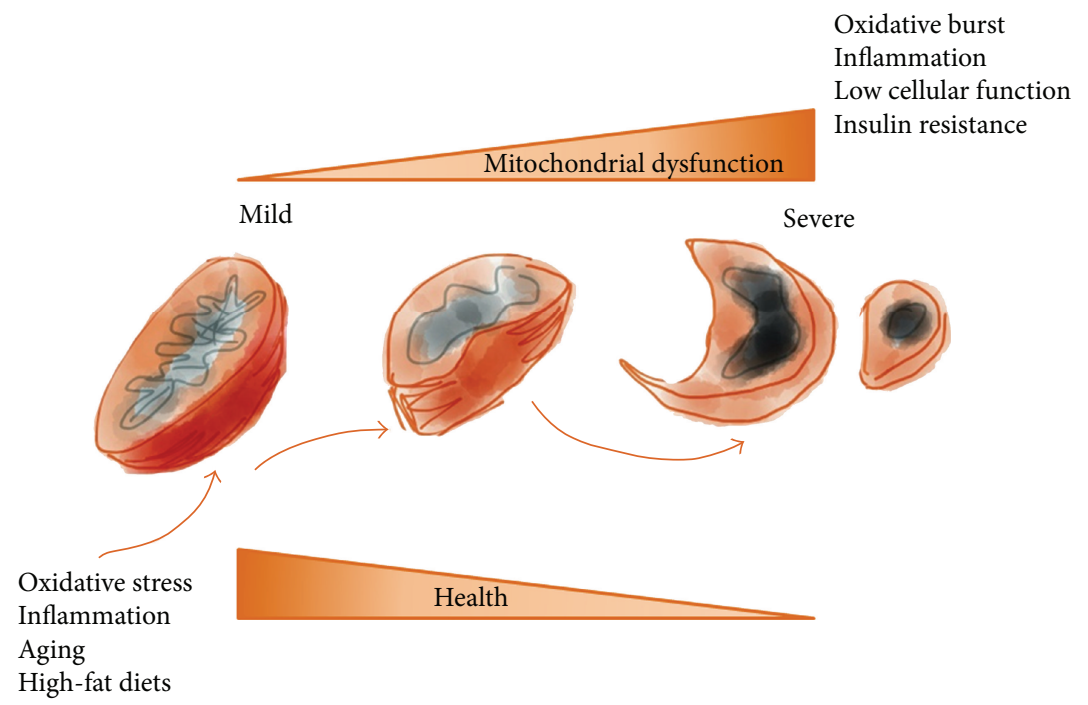

FIGURE 1: Mutations in mitochondrial DNA are accompanied by different disease-suggestive phenotypes (myopathies, neuropathies, diabetes, and signs of reduced lifespan and premature aging). Severe mitochondrial dysfunction triggers a high level of oxidative and inflammatory damage, impairs tissue function, and promotes age-related diseases.
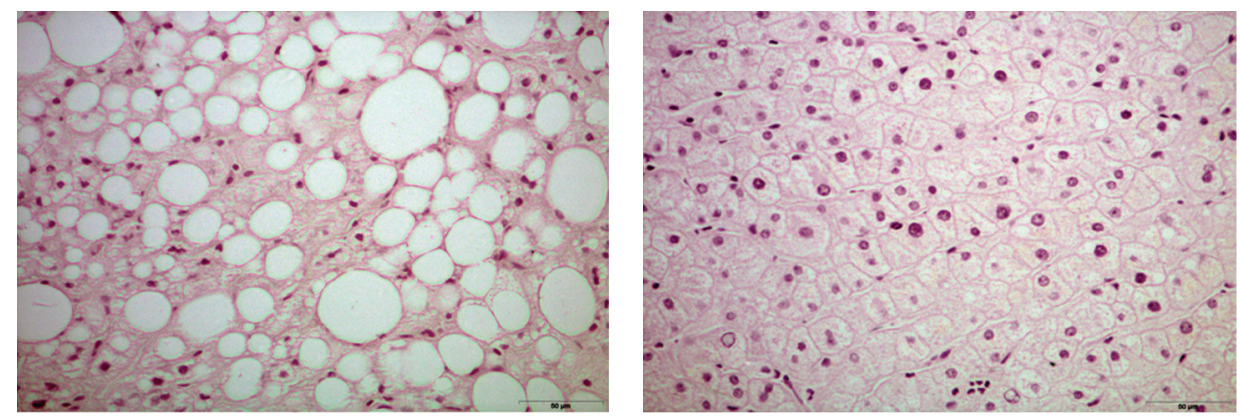

(a)
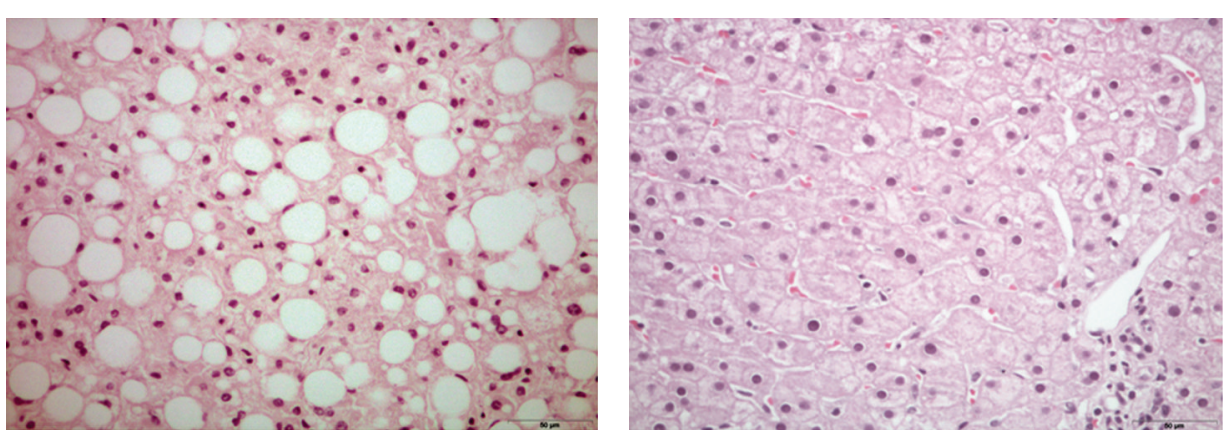

(b)

Figure 2: Clinically, it is evident that, in severe obesity, (a) the presence of liver steatosis may vary from more than $80 \%$ to less than $5 \%$ of patients. Conversely, in most obese patients with some degree of liver steatosis (b), this condition disappeared in a relatively brief period of time after significant weight loss due to bariatric surgery.

are increased, and M2 or "alternatively activated" ATMs are decreased in the adipose tissues of both obese mice and obese humans, as discussed below $[46,47]$.

It is frequently assumed that, in contrast to hormones, chemokines influence cellular activities in an autocrine or paracrine fashion. However, chemokines may be relevant effectors in chronic systemic inflammation as the confinement of these molecules to well-defined environments is unlikely. Specifically, alterations in plasma MCP-1 concentrations in metabolic disease states, the presence of circulating chemokine reservoirs, recent evidence of novel mechanisms of action, and certain unexplained responses associated with 


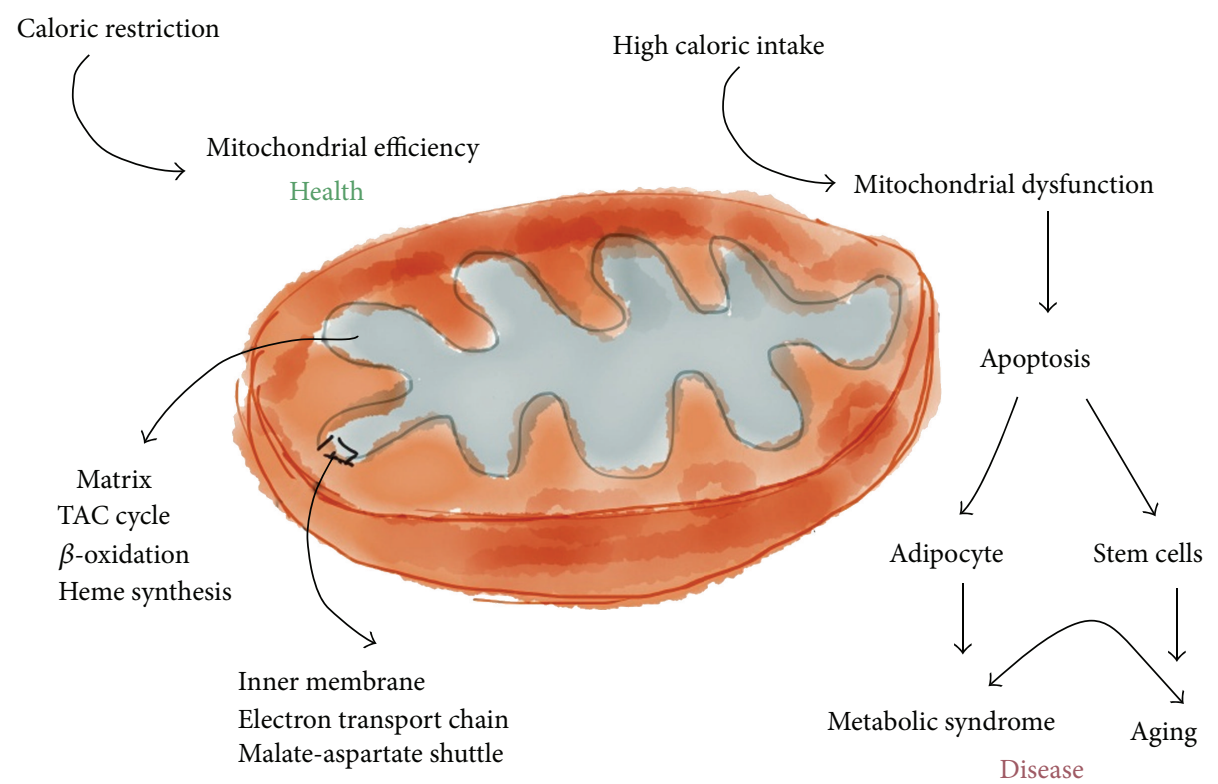

Figure 3: The mitochondrial matrix hosts the mitochondrial metabolic pathways (TAC cycle, $\beta$-oxidation, and haem synthesis), and the inner membrane contains the electron transport chain complexes and ATP synthase. Exchange carriers such as the malate-aspartate shuttle are also essential. Under caloric restriction, the mitochondrion achieves the highest efficiency, and high caloric intake produces dysfunction and a consequent increase in apoptosis, which promotes metabolic syndrome and age-related diseases.



FIGURE 4: Schematic and abridged representation of the multiple roles of mitochondria in cellular processes that are associated with the pathogenesis of the more prevalent diseases.

metabolic disturbances suggest that MCP-1 might have a systemic role in metabolic regulation [48-50]. How and when obesity might initiate an inflammatory response remains controversial, but the underlying mechanism likely depends on the activation of the c-Jun $\mathrm{N}$-terminal kinase (JNK) in insulin-sensitive tissues, as JNK is likely the principal mechanism through which inflammatory signals interfere with insulin activity [7].
ER stress responses and mitochondrial defects are also linked to the mTOR pathway, discussed below, which is essential for the regulation of numerous processes, including the cell cycle, energy metabolism, the immune response, and autophagy. Therefore, the specific cellular changes associated with metabolic alterations, particularly mitochondrial dysfunction, require further attention.

\section{Mitochondria: Bioenergy Couples Metabolism, Oxidation, and Inflammation}

Mitochondria are essential organelles that, among other functions, supply the cell with ATP through oxidative phosphorylation, synthesise key molecules, and buffer calcium gradients; however, they are also a source of free radicals (Figures 1, 3, and 4). It is not surprising that mitochondrial health is tightly regulated and associated with the homeostasis and aging of the organism. Within these processes, the antagonistic and balanced activities of the fusion and fission machineries constantly provide adequate responses to events caused by inflammation (Figure 5) [23, 50-54]. A shift towards fusion favours the generation of interconnected mitochondria, which contribute to the dissipation and rapid provision of energy. A shift towards fission results in numerous mitochondrial fragments. Apparently, the mixing of the matrix and the inner membrane allows the respiratory machinery components to cooperate most efficiently. Furthermore, fusion maximises ATP synthesis. In quiescent cells, mitochondria are frequently present as numerous morphologically and functionally distinct small spheres or short rods $[51,55,56]$. Upon the exposure of cells to stress, fusion optimises mitochondrial function and 


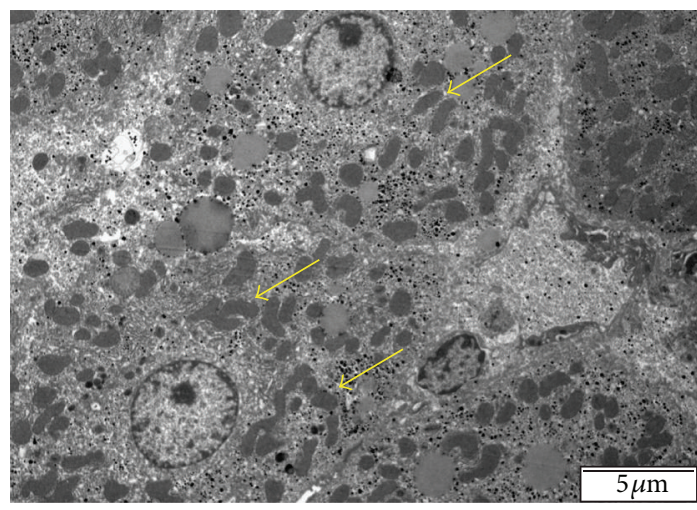

(a)



(b)

FiguRE 5: Mitochondrial fusion (a) and fission (b) processes in the liver (arrows). Mitochondrial morphology is basically controlled by metabolism and inflammation, and each change in morphology is mediated by large guanosine triphosphatases of the dynamin family, consistent with a model in which the capacity for oxidative phosphorylation is maximised under stressful conditions.



FIGURE 6: The complete elimination of mitochondria by autophagy (arrow) is a process linked to mitochondrial fission and fusion. Mitochondria also employ quality-control proteases to eliminate damaged molecules through the transcriptional induction of chaperones or the ubiquitin proteasome quality-control pathway.

plays a beneficial role in the maintenance of long-term bioenergetics capacities. In contrast, the mitochondrial fission machinery contributes to the elimination of irreversibly damaged mitochondria through autophagy [55-58]. This process, also called mitophagy, is extremely important under both physiological and pathological conditions (Figure 6). A detailed discussion of the importance of mitophagy is beyond the scope of this review; however, as an example of its importance, recall that amino acids are not stored in the body but are instead mobilised by proteolysis under conditions such as starvation, reduced physical activity, and disease [59]. Furthermore, intense exercise may modulate hepatic metabolism through similar mechanisms [60]. More recently, the mitochondrial E3 ubiquitin protein ligase 1 (Mul 1) was identified as a key protein that promotes mitophagy and skeletal muscle loss [61]. Mitochondrial fission per se triggers organelle dysfunction and muscle loss. The opposite is observed when mitochondrial fission is inhibited. The same authors [61] also demonstrated that the overexpression of Forkhead box $\mathrm{O} 3$ (FoxO3) induces mitochondrial disruption via mitophagy.

Therefore, it is not surprising that mitochondrial diseases often have an associated metabolic component, and consequently mitochondrial defects are expected in inflammation, aging, and other energy-dependent disturbances [58, 62]. In such disturbances, cellular oxidative damage caused by the generation of reactive oxygen species (ROS) that exceed the natural antioxidant activity is likely an initiating factor in inflammation and aging $[63,64]$. Several potential therapeutic approaches are currently available to slow down age-related functional declines [65], including antioxidant treatments [66]; however, the effectiveness of existing antioxidants is likely suboptimal because these antioxidants are not selective for mitochondria [67]. However, recent experiments with a mitochondria-targeted antioxidant have been successful in animal models [67]. Similar assumptions can be made for endothelial cells, in which oxidation and the accompanying inflammation are recognised factors for atherosclerosis. Oxidative stress, which is mainly derived from mitochondrial dysfunction, decreases NO synthesis, contributes to hypertension, upregulates the secretion of adhesion molecules and inflammatory cytokines, and is responsible for the oxidation of low-density lipoproteins [68, 69].

Defective mitochondrial function in muscle tissues leads to reduced fatty acid oxidation and the inhibition of glucose transport, indicating that insulin-stimulated glucose transport is reduced. This is a hallmark of insulin resistance and type 2 diabetes. The chronic production of excess ROS and inflammation result in mitochondrial dysfunction potentially inducing lipid accumulation in these tissues and the endless vicious cycle of insulin resistance [70-74]. Mitochondrial ROS have also been related to the increased activity of uncoupling proteins (UCP), which uncouple ATP synthesis from electron transport. UCP activity leads to heat generation without ATP production, and long-term reductions in ATP 
levels affect cellular insulin signalling. The roles of the UCPs and the metabolically relevant differences between brown and white adipose tissues were reviewed recently [75-77].

The mitochondria of obese individuals are different from those of lean individuals. Alterations in mitochondrial morphology, impaired mitochondrial bioenergetics, increased mitochondrial lipid peroxides, decreased ATP content, and mitochondrial dysfunction further increase the risks of developing metabolic complications [78, 79]. In comparison to those of lean individuals, mitochondria in obese individuals have lower energy-generating capacities, less clearly defined inner membranes, and reduced fatty acid oxidation. These differences might promote the development and progression of obesity and might also have therapeutic implications [80, 81]. Impaired mitochondrial function could account for the insulin resistance that is closely associated with increased lipid content in the muscles of patients with type 2 diabetes. Altered mitochondrial function is the major factor that leads to increased muscular lipid accumulation and decreased insulin sensitivity [80, 81]. More recently, a model was created in which the amount of mitochondrial activity in adipocytes and hepatocytes can be altered based on the properties of the mitochondrial protein mitoNEET, which is located at the outer membrane [70]. Despite the prevalence of obesity in this model, mitoNEET overexpression during periods of high caloric intake resulted in systemwide improvements in insulin sensitivity, thereby providing a model of a "metabolically healthy" obese state with minimal tissue lipotoxicity that is similar to the clinically observed condition [82]. Alterations in mitoNEET expression might modulate ROS concentrations and mitochondrial iron transport into the matrix $[70,82,83]$. The mitochondrial fusion protein mitofusin-2 (Mfn-2), another useful protein in studies of mitochondrial dysfunction, regulates cellular metabolism and controls mitochondrial metabolism. In cultured cells, mitochondrial metabolism was activated in Mfn-2 gainof-function experiments, whereas Mfn-2 loss-of-function reduced glucose oxidation, mitochondrial membrane potential, oxygen consumption, and mitochondrial proton leakage [84]. It is defective in the muscles of obese and type 2 diabetes patients in which mitochondrial size is reduced [71].

Therefore, a detailed characterisation of the proteins involved in mitochondrial fusion and fission and studies of the mechanisms that regulate these two processes are relevant to human pathology and might have a great therapeutic potential to improve metabolism and to decrease the generation of oxidative stress and excessive inflammatory response [85].

\section{Is There a Link between Mitochondria and Nutrient Availability? The Possible Roles of Inflammation and Apoptosis}

Apoptosis is another basic process to consider in metabolic diseases. Excess food intake leads to mitochondrial dysfunction and higher apoptotic susceptibility. Mitochondria specialise in energy production and cell killing. Only 13 proteins are encoded by the mitochondrial DNA, a circular molecule of $16 \mathrm{~Kb}$. The remaining necessary proteins are encoded in the nuclear DNA [86]. Mitochondria are composed of outer and inner specialised membranes that define two separate components, the matrix and the intermembrane space [87]. Mitochondria regulate apoptosis in response to cellular stress signals and determine whether cells live or die [88]. Thus, it is conceivable that the availability or ingestion of nutrients could be a main candidate in the regulation of cell death and that mitochondria could have been selected as a nutrient sensor and effector. This could explain the influence of apoptosis-related proteins on mitochondrial respiration [89].

A common laboratory finding is that the morphology of the mitochondria changes when mice are supplied with a high-fat diet (Figure 7) and that optimal mitochondrial performance is achieved under conditions of calorie restriction. Excess food intake impairs respiratory capacities, likely through mTOR, and increases the susceptibility of the cell to apoptosis and additional stress [90, 91]. Of note, apoptotic protein levels are increased in the adipocytes of obese humans, and the depletion of proapoptotic proteins protects against liver steatosis and insulin resistance in mice fed a high-fat, high-cholesterol diet [92]. These conditions are relevant to the development of metabolic syndrome, as nutritional imbalances in Western diets lead to mitochondrial dysfunction and higher susceptibilities to inflammation, apoptosis, and aging [22].

\section{AMP-Activated Protein Kinase (AMPK) Not Only Influences Metabolism in Adipocytes but Also Suppresses the Proinflammatory Environment}

AMPK has anti-inflammatory actions that are independent of its effects on glucose and lipid metabolism [93]. The action of AMPK is not necessarily identical in all tissues. In adipose tissues, the role of AMPK is largely unknown because laboratory techniques to explore the action of this kinase in terminally differentiated adipocytes have not been fully established. Several agents have been used to activate AMPK experimentally, including AICAR ( 5 -aminoimidazole-4-carboxamide ribonucleoside), metformin, rosiglitazone, resveratrol and other polyphenols, statins, and several adipocytokines. In adipocytes, AMPK appears to increase the insulin-stimulated uptake of glucose, likely by increasing the expression of GLUT4, yet inhibits glucose metabolism [94]. Studies of the effects of AMPK on lipolysis in adipocytes have been controversial; some authors have reported an antilipolytic effect, while others have suggested that AMPK stimulates lipolysis $[95,96]$. However, the activation of AMPK by metformin in human adipose tissues increases the phosphorylation of acetyl-CoA carboxylase (ACC) and decreases the expression of lipogenic genes, leading to reductions in malonyl-CoA, which is the precursor for fatty acid synthesis; malonyl-CoA also regulates fatty acid oxidation through the inhibition of carnitine palmitoyl-transferase 1, the rate-limiting enzyme for fatty acid entry into the mitochondria [97, 98]. Adipose tissue secretes adipocytokines, which influence metabolic and inflammatory pathways through the recruitment of 




(a)

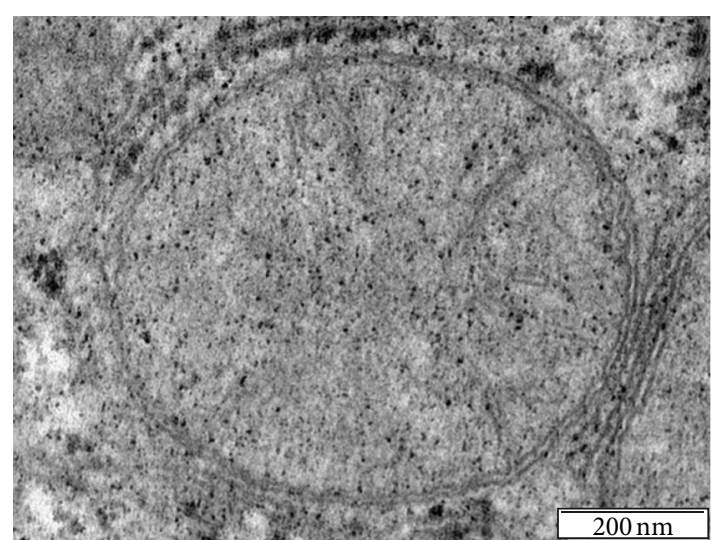

(b)

FIGURE 7: The nutrient availability of food in "natural" conditions for mice is likely low and near the condition known as calorie restriction. In the laboratory, however, mice are usually fed ad libitum, and certain biases cannot be discarded. However, mitochondria from mice fed a chow diet (a) display rapid morphological changes when mice are fed with high-fat diets (b).

macrophages and the consequent transition from the M2 state to M1 [7, 41]. These actions contribute to the development of disease (Figure 8). Conversely, adiponectin has been reported to induce adipose macrophages to switch to the antiinflammatory M2 state [99]. AMPK is anti-inflammatory, as it inhibits the synthesis of proinflammatory cytokines and promotes the expression of IL-10 in macrophages; adiponectin and leptin levels may also be regulated by AMPK [100] (Figure 8). Finally, brown adipocytes contain high numbers of mitochondria that express UCP1, which permit thermogenesis. Exposure to cold temperatures stimulates AMPK and may play a role in the differentiation of fatty oxidising brown adipose tissue, thus leading to greater energy expenditure [101]. Therefore, we hypothesise that the chronic manipulation of the AMPK/mechanistic target of rapamycin (mTOR) pathway might represent a therapeutic approach for preventing noncommunicable diseases (Figure 8). Metformin, along with salicylate, polyphenols, and rapamycin, has a long history of safe and effective use, but other modulators are currently under development and will likely permit the design of tissue-specific activators of this pathway.

\section{Metformin and/or Rapamycin and Plant-Derived Polyphenols: An Apparent Treatment of Choice for Metabolic Syndrome and Obesity-Related Complications?}

The first therapeutic approaches to metabolic disturbances are reduced caloric ingestion and increased physical activity. The effects are based mainly on weight reduction, but usefulness in other common complications remains incompletely explored [102]. Bariatric surgery is also effective, even in "metabolically healthy" patients $[103,104]$. The effectiveness of surgery for the treatment of metabolic disturbances is surprisingly higher than expected, and mechanisms associated with surgical effects are not completely understood.
Insulin resistance and mitochondrial dysfunction appear to be the most significant alternative therapeutic targets. Metabolic abnormalities are associated with inflammation. Normally, glycolysis yields pyruvate, which is further oxidised in the mitochondria. When oxygen becomes limiting, mitochondrial oxidative metabolism is restricted. The induction of an inflammatory response is an energy-intensive process, and the involved cells rapidly switch from resting to highly active states. This is observed in diseases such as cancer, atherosclerosis, or autoimmune diseases, and mechanistic insights suggest the common involvement of the transcription factor hypoxia-inducible factor $1 \alpha$, AMPK, and the mTOR pathway. In addition, the activation of sirtuins, which act as NAD+ sensors that connect nutrition and metabolism to chromatin structure, is anti-inflammatory [105] (Figure 8).

The use of metformin, an AMPK activator used extensively to treat type 2 diabetes, has been indicated for other metabolic conditions based on the rationale that insulinsensitising agents might be effective [106], and the mode of action of metformin has guided our own experiments on cancer, aging, and viral infection $[65,107,108]$. We have shown that the beneficial effects of this biguanide class drug, which was initially obtained from Galega officinalis, are universal in patients with metabolic complications and negligible in patients without such complications. The primary effect is thought to be the suppression of hepatic glucose production and hepatic lipogenesis [109]. Metformin activates AMPK in hepatocytes, resulting in the phosphorylation and inactivation of ACA, a rate-limiting enzyme in lipogenesis [110], and theoretically might be useful and safe in the treatment of NAFLD [111]. Surprisingly, the beneficial clinical effects seem to be limited, despite the effects of metformin on insulin resistance, most likely because long-term treatment is an absolute requirement for the prevention of progressive disease. Our own current experiments in animal models suggest new insights into this phenomenon. Metformin activates AMPK, but AMPK deficiency does not abolish 


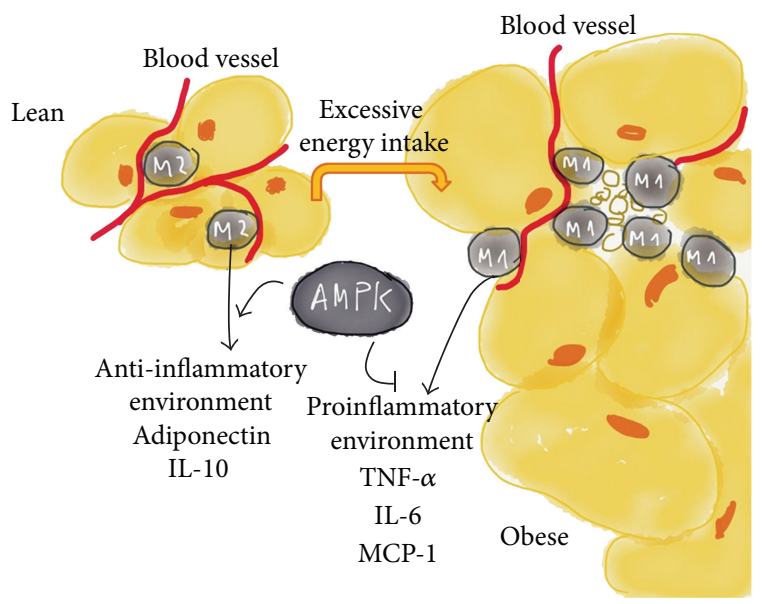

(a)

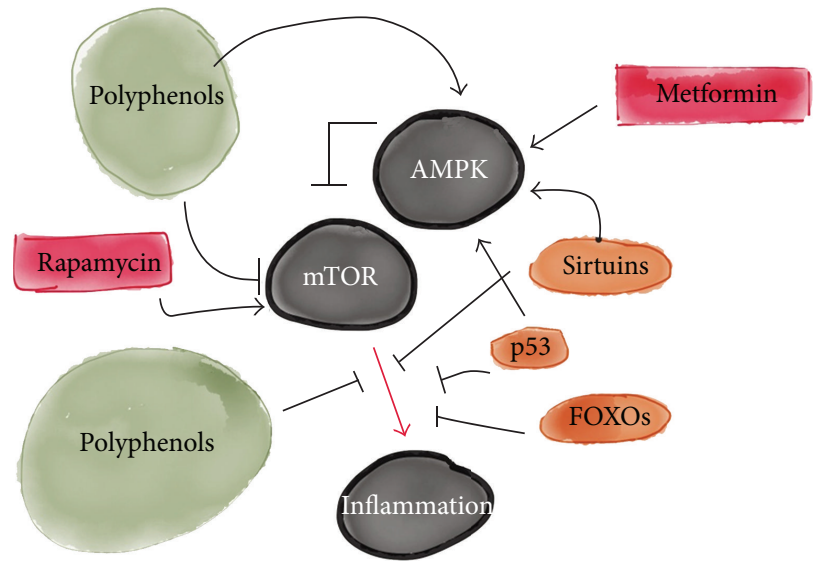

(b)

FIGURE 8: Activation of AMPK in macrophages promotes the switch from a proinflammatory to an anti-inflammatory phenotype by inducing a shift from glycolysis towards mitochondrial oxidative metabolism. In obesity, there may be a shift towards proinflammatory states, whereas in dietary restriction the balance may shift towards anti-inflammatory phenotypes through the activation of AMPK (a). The activation of AMPK implies the inhibition of mTOR, and several compounds are known to regulate this pathway (b). The inhibition of mTOR extends lifespan in model organisms and confers protection against a growing list of age-related pathologies. Several characterised inhibitors are already clinically approved, and others are under development.

the effects of metformin on hepatic glucose production, indicating that the role of AMPK is dispensable, as indicated previously [112]. This suggests that the overall effect of metformin is mediated through actions on mitochondrial function through decreases in the hepatic energy state and intracellular ATP content. Other studies suggest that metformin inhibits Complex I of the mitochondrial respiratory chain, but the exact mechanisms and pathways involved are unclear [113]. Sirtuin 3 (SIRT 3), a member of the family of nicotinamide adenine dinucleotide (NAD+) dependent deacetylase proteins, is a crucial regulator of mitochondrial function that controls the global acetylation of the organelle (all sirtuins regulate energy production and the cell cycle; Figure 8). SIRT3 induces the activity of Complex I and promotes oxidative phosphorylation. In SIRT3 knockout mice, mitochondrial proteins are hyperacetylated, and cellular ATP levels are reduced, effects that are aggravated by fasting [114]. As a complement, peroxisome proliferator-activated receptor gamma coactivator 1-alpha induces the expression of SIRT3 in the liver [115]. Therefore, mitochondrial function appears to be the key target of metformin; reductions in ATP production may mediate the hepatic and antihyperglycemic actions of the drug and downregulate SIRT3 expression [116]. However, metformin distinctively regulates the expression of different sirtuin family members [117, 118]. In summary, metformin acts against both insulin resistance and mitochondrial dysfunction and is currently an attractive candidate agent of choice in the management of metabolic disorders. We have recently reviewed this complex scenario and found the following: (1) the unique ability of metformin to activate AMPK while leading to the increased utilisation of energy occurs because metformin inhibits AMP deaminase; and (2) in metabolic tissues, metformin can inhibit cell growth by functionally mimicking the effects of a multitargeted antifolate [119].

Based on these and other findings, we have also demonstrated that plant-derived phenolic compounds interact with numerous targets and multiple deregulated signalling pathways that may be useful in the management of metabolic conditions [120-123]. The proposed mechanisms are direct antioxidant activity, attenuation of endoplasmic reticulum stress, blockade of proinflammatory cytokines, and blockade of transcription factors related to metabolic diseases [120]. Most polyphenols modulate oxidative stress and inflammatory responses through relevant actions in the process of macrophage recruitment. Interactions between the chemokine/cytokine network and bioenergetics, likely through the mTOR pathway, may also represent potential mechanisms for the prevention of metabolic disturbances [121]. Moreover, polyphenols attenuate the metabolic effects of high-fat, high-cholesterol diets when administered continuously at high doses, and we have described beneficial actions associated with the expression of selected microRNAs [122].

Inflammation lies at the heart of many diseases because the entire body is under metabolic stress, which induces symptoms and causes morbidity. Targeting altered metabolic pathways in inflammation may enhance our understanding of disease pathogenesis and point the way to new therapies. As mentioned, metformin, polyphenols, AICAR, salicylates, and corticoids all activate the AMPK/mTOR pathway. New compounds such as A-769662 are under scrutiny. Finally, rapamycin, which is also known as sirolimus and was first isolated from Streptomyces hygroscopicus, and several derivative compounds, including everolimus, temsirolimus, ridaforolimus, umirolimus, and zotarolimus, have been approved for a variety of uses, including posttransplantation 
therapy, the prevention of restenosis following angioplasty, and as a treatment for certain forms of cancer. Drugs that inhibit the mTOR pathway could one day be used widely to slow aging and reduce age-related pathologies in humans [124]. The development of chemical inhibitors of mTOR, as well as drugs that target other components of the mTOR pathway, promises to aid research greatly while also providing drugs with potential therapeutic value.

\section{Perspectives and Implications}

Obesity, metabolic alterations, and age-related diseases are complex conditions that require a multifaceted approach that includes action on both the chemokine network and energy metabolism [123, 125]. The underlying mechanisms are far from being understood [126] although the association between obesity and insulin resistance seems to be well substantiated. However, obesity is not distributed normally throughout the population, and type 2 diabetes mellitus is not associated closely with increased body weight; also, the relationship with noncommunicable diseases is not straightforward. A working hypothesis is that adipose tissue has a limited maximum capacity to increase in mass. Once the adipose tissue has reached the expansion limit, fat is deposited in the liver and muscle tissues and causes insulin resistance. This process is also associated with the activation of macrophages, oxidative stress, and inflammation which produce cytokines that have negative effects on insulin sensitivity, induce the secretion of adipokines that cause insulin resistance, and suppress those that promote insulin sensitivity. However, a host of other mechanisms must be involved because metabolic responses are different among patients with maximum adipose tissue expansion. A more popular and recent hypothesis suggests a differential effect of lipophagy, which implies a tissue-selective autophagy with cellular consequences from the mobilisation of intracellular lipids. Defective lipophagy is linked to fatty liver tissues and obesity and might be the basis for age-related metabolic syndrome [127]. Increased adipose tissue autophagy may be responsible for more efficient storage. Autophagy also affects metabolism, oxidation, and proinflammatory cytokine production. Very recent evidence suggests that autophagy is increased in the adipose tissues of obese patients [128]. Inexpensive and well-tolerated molecules such as chloroquine, metformin, and polyphenols already exist and could be used to fine-tune the metabolic alterations derived from an excess of energy and, more specifically, to modulate autophagy in the liver. Whether these therapies will dampen the genetic expression of factors that affect the development of noncommunicable diseases remains to be ascertained.

\section{Acknowledgments}

The Unitat de Recerca Biomèdica is currently being supported by Grants from the Fondo de Investigación Sanitaria (FIS PI08/1032, PI11/00130). E. Rodríguez-Gallego is the recipient of a fellowship from the Generalitat de Catalunya (2012FI_B 00389), and M. Riera-Borrull is the recipient of a fellowship from the Universitat Rovira i Virgili (2010PFRURV-B2-58).

\section{References}

[1] K. Strong, C. Mathers, S. Leeder, and R. Beaglehole, "Preventing chronic diseases: how many lives can we save?" The Lancet, vol. 366, no. 9496, pp. 1578-1582, 2005.

[2] M. Cecchini, F. Sassi, J. A. Lauer, Y. Y. Lee, V. GuajardoBarron, and D. Chisholm, "Tackling of unhealthy diets, physical inactivity, and obesity: health effects and cost-effectiveness," The Lancet, vol. 376, no. 9754, pp. 1775-1784, 2010.

[3] T. A. Gaziano, G. Galea, and K. S. Reddy, "Scaling up interventions for chronic disease prevention: the evidence," The Lancet, vol. 370, no. 9603, pp. 1939-1946, 2007.

[4] E. A. H. Sims, "Are there persons who are obese, but metabolically healthy?" Metabolism, vol. 50, no. 12, pp. 1499-1504, 2001.

[5] N. Stefan, K. Kantartzis, J. Machann et al., "Identification and characterization of metabolically benign obesity in humans," Archives of Internal Medicine, vol. 168, no. 15, pp. 1609-1616, 2008.

[6] G. Iacobellis, M. C. Ribaudo, A. Zappaterreno, C. V. Iannucci, and F. Leonetti, "Prevalence of uncomplicated obesity in an Italian obese population," Obesity Research, vol. 13, no. 6, pp. 1116-1122, 2005.

[7] A. Rull, J. Camps, C. Alonso-Villaverde, and J. Joven, "Insulin resistance, inflammation, and obesity: role of monocyte chemoattractant protein-1 (orCCL2) in the regulation of metabolism," Mediators of Inflammation, vol. 2010, Article ID 326580, 11 pages, 2010.

[8] J. B. Meigs, I. Lipinska, S. Kathiresan et al., "Visceral and subcutaneous adipose tissue volumes are cross-sectionally related to markers of inflammation and oxidative stress: the framingham heart study," Circulation, vol. 116, no. 11, pp. 1234-1241, 2007.

[9] D. Dowell and T. A. Farley, "Prevention of non-communicable diseases in New York City," The Lancet, vol. 380, no. 9855, pp. 1787-1792, 2012.

[10] D. M. Arduíno, A. R. Esteves, and S. M. Cardoso, "Mitochondria drive autophagy pathology via microtubule disassembly: a new hypothesis for Parkinson disease," Autophagy, vol. 9, no. 1, pp. 112-114, 2013.

[11] H. Kumar, H. W. Lim, S. V. More et al., "The role of free radicals in the aging brain and Parkinson's disease: convergence and parallelism," International Journal of Molecular Science, vol. 13, no. 8, pp. 10478-10504, 2012.

[12] G. Medina-Gómez, "Mitochondria and endocrine function of adipose tissue," Best Practice \& Research Clinical Endocrinology \& Metabolism, vol. 26, no. 6, pp. 791-804, 2012.

[13] G. Pagano, G. Castello, and F. V. Pallardó, "Sjøgren's syndromeassociated oxidative stress and mitochondrial dysfunction: prospects for chemoprevention trials," Free Radical Research, vol. 47, no. 2, pp. 71-73, 2013.

[14] J. Ouyang, M. Wu, C. Huang, L. Cao, and G. Li, "Overexpression of oxidored-nitro domain containing protein 1 inhibits human nasopharyngeal carcinoma and cervical cancer cell proliferation and induces apoptosis: involvement of mitochondrial apoptotic pathways," Oncology Reports, vol. 29, no. 1, pp. 79-86, 2013.

[15] L. D. Osellame, T. S. Blacker, and M. R. Duchen, "Cellular and molecular mechanisms of mitochondrial function," Best Practice \& Research Clinical Endocrinology \& Metabolism, vol. 26, no. 6, pp. 711-723, 2012. 
[16] I. Enache, A. L. Charles, J. Bouitbir et al., "Skeletal muscle mitochondrial dysfunction precedes right ventricular impairment in experimental pulmonary hypertension," Molecular and Cellular Biochemistry, vol. 373, no. 1-2, pp. 161-170, 2013.

[17] L. W. Chol, "Metabolic syndrome," Singapore Medical Journal, vol. 52, no. 11, pp. 779-785, 2011.

[18] M. R. Souza, F. D. Mde, J. E. Medeiros-Filho, and M. S. Araújo, "Metabolic syndrome and risk factors for non-alcoholic fatty liver disease," Arquivos de Gastroenterologia, vol. 49, no. 1, pp. 89-96, 2012.

[19] M. S. Mirza, "Obesity, visceral fat and NAFLD: querying the role of adipokines in the progression of nonalcoholic fatty liver disease," ISRN Gastroenterology, vol. 2011, Article ID 592404, 11 pages, 2011.

[20] G. Tarantino, S. Savastano, and A. Colao, "Hepatic steatosis, low-grade chronic inflammation and hormone/growth factor/adipokine imbalance," World Journal of Gastroenterology, vol. 16, no. 38, pp. 4773-4783, 2010.

[21] C. Vernochet and C. R. Kahn, "Mitochondria, obesity and aging," Aging, vol. 4, no. 12, pp. 1-2, 2012.

[22] F. Pintus, G. Floris, and A. Rufini, "Nutrient availability links mitocondria, apoptosis and obesity," Aging, vol. 4, no. 11, pp. 18, 2012.

[23] M. M. Rogge, "The role of impaired mitochondrial lipid oxidation in obesity," Biological Research for Nursing, vol. 10, no. 4, pp. 356-373, 2009.

[24] M. Vinaixa, M. A. Rodríguez, A. Rull et al., "Metabolomic assessment of the effect of dietary cholesterol in the progressive development of fatty liver disease," Journal of Proteome Research, vol. 9, no. 5, pp. 2527-2538, 2010.

[25] A. Rull, M. Vinaixa, M. Ángel Rodríguez et al., "Metabolic phenotyping of genetically modified mice: an NMR metabonomic approach," Biochimie, vol. 91, no. 8, pp. 1053-1057, 2009.

[26] J. Joven, A. Rull, N. Ferré et al., “The results in rodent models of atherosclerosis are not interchangeable. The influence of diet and strain," Atherosclerosis, vol. 195, no. 2, pp. e85-e92, 2007.

[27] M. Tous, N. Ferré, J. Camps, F. Riu, and J. Joven, "Feeding apolipoprotein E-knockout mice with cholesterol and fat enriched diets may be a model of non-alcoholic steatohepatitis," Molecular and Cellular Biochemistry, vol. 268, no. 1-2, pp. 53-58, 2005.

[28] P. Lindström, " $\beta$-cell function in obese-hyperglycemic mice [ob/ob mice]," Advances in Experimental Medicine and Biology, vol. 654, pp. 463-477, 2010.

[29] S. de Ferranti and D. Mozaffarian, "The perfect storm: obesity, adipocyte dysfunction, and metabolic consequences," Clinical Chemistry, vol. 54, no. 6, pp. 945-955, 2008.

[30] L. Xu, X. Ma, B. Cui, X. Li, G. Ning, and S. Wang, "Selection of reference genes for qRT-PCR in high fat diet-induced hepatic steatosis mice model," Molecular Biotechnology, vol. 48, no. 3, pp. 255-262, 2011.

[31] M. V. Machado, J. Coutinho, F. Carepa, A. Costa, H. Proença, and H. Cortez-Pinto, "How adiponectin, leptin, and ghrelin orchestrate together and correlate with the severity of nonalcoholic fatty liver disease," European Journal of Gastroenterology and Hepatology, vol. 24, no. 10, pp. 1166-1172, 2012.

[32] J. C. Cohen, J. D. Horton, and H. H. Hobbs, "Human fatty liver disease: old questions and new insights," Science, vol. 332, no. 6037, pp. 1519-1523, 2011.

[33] M. J. Pagliassotti, "Endoplasmic reticulum stress in nonalcoholic fatty liver disease," Annual Review of Nutrition, vol. 32, pp. 17-33, 2012.
[34] J. V. Neel, "Diabetes mellitus a "thrifty" genotype rendered detrimental by 'progress'?" The American Journal of Human Genetics, vol. 14, pp. 352-353, 1962.

[35] J. V. Neel, "Update to 'The study of natural selection in primitive and civilized human populations," Human Biology, vol. 61, no. 5-6, pp. 811-823, 1989.

[36] A. R. Frisancho, "Reduced rate of fat oxidation: a metabolic pathway to obesity in the developing nations," The American Journal of Human Biology, vol. 15, no. 4, pp. 522-532, 2003.

[37] J. R. Speakman, "A novel non-adaptive scenario explaining the genetic pre-disposition to obesity: the "predation release" hypothesis," Cell Metabolism, vol. 6, no. 1, pp. 5-12, 2007.

[38] J. R. Speakman and S. O'Rahilly, "Fat: an evolving issue," Disease Models and Mechanisms, vol. 5, no. 5, pp. 569-573, 2012.

[39] B. Rius, C. López-Vicario, A. González-Périz et al., "Resolution of inflammation in obesity-induced liver disease," Frontiers in Immunology, vol. 3, article 257, 2012.

[40] A. Paul, L. Calleja, J. Camps et al., "The continuous administration of aspirin attenuates atherosclerosis in apolipoprotein Edeficient mice," Life Sciences, vol. 68, no. 4, pp. 457-465, 2000.

[41] M. Tous, N. Ferré, A. Rull et al., "Dietary cholesterol and differential monocyte chemoattractant protein-1 gene expression in aorta and liver of apo E-deficient mice," Biochemical and Biophysical Research Communications, vol. 340, no. 4, pp. 10781084, 2006.

[42] L. Masana, M. Camprubi, P. Sarda, R. Sola, J. Joven, and P. R. Turner, "The mediterranean-type diet: is there a need for further modification?" The American Journal of Clinical Nutrition, vol. 53, no. 4, pp. 886-889, 1991.

[43] A. P. Rolo, J. S. Teodoro, and C. M. Palmeira, "Role of oxidative stress in the pathogenesis of nonalcoholic steatohepatitis," Free Radical Biology and Medicine, vol. 52, no. 1, pp. 59-69, 2012.

[44] B. Mlinar and J. Marc, "New insights into adipose tissue dysfunction in insulin resistance," Clinical Chemistry and Laboratory Medicine, vol. 29, no. 12, pp. 1925-1935, 2011.

[45] C. N. Lumeng, J. L. Bodzin, and A. R. Saltiel, "Obesity induces a phenotypic switch in adipose tissue macrophage polarization," Journal of Clinical Investigation, vol. 117, no. 1, pp. 175-184, 2007.

[46] M. E. Shaul, G. Bennett, K. J. Strissel, A. S. Greenberg, and M. S. Obin, "Dynamic, M2-like remodeling phenotypes of $\mathrm{CD}_{11 \mathrm{c}^{+}}$adipose tissue macrophages during high-fat dietinduced obesity in mice," Diabetes, vol. 59, no. 5, pp. 1171-1181, 2010.

[47] J. M. Wentworth, G. Naselli, W. A. Brown et al., "Proinflammatory $\mathrm{CD}_{11 c^{+}} \mathrm{CD} 206^{+}$adipose tissue macrophages are associated with insulin resistance in human obesity," Diabetes, vol. 59, no. 7, pp. 1648-1656, 2010.

[48] A. Rull, R. Beltrán-Debón, G. Aragonès et al., "Expression of cytokine genes in the aorta is altered by the deficiency in MCP1: effect of a high-fat, high-cholesterol diet," Cytokine, vol. 50, no. 2, pp. 121-128, 2010.

[49] M. L. Batista, S. B. Peres, M. E. McDonald et al., "Adipose tissue inflammation and cancer cachexia: possible role of nuclear transcription factors," Cytokine, vol. 57, no. 1, pp. 9-16, 2012.

[50] A. Rull, J. C. Escolà-Gil, J. Julve et al., "Deficiency in monocyte chemoattractant protein-1 modifies lipid and glucose metabolism," Experimental and Molecular Pathology, vol. 83, no. 3, pp. 361-366, 2007.

[51] B. Coll, C. Alonso-Villaverde, and J. Joven, "Monocyte chemoattractant protein-1 and atherosclerosis: Is there room for an additional biomarker?" Clinica Chimica Acta, vol. 383, no. 1-2, pp. 21-29, 2007. 
[52] B. Westermann, "Mitochondrial fusion and fission in cell life and death," Nature Reviews Molecular Cell Biology, vol. 11, no. 12, pp. 872-884, 2010.

[53] D. C. Chan, "Mitochondrial fusion and fission in mammals," Annual Review of Cell and Developmental Biology, vol. 22, pp. 79-99, 2006.

[54] D. H. Margineantu, W. G. Cox, L. Sundell, S. W. Sherwood, J. M. Beechem, and R. A. Capaldi, "Cell cycle dependent morphology changes and associated mitochondrial DNA redistribution in mitochondria of human cell lines," Mitochondrion, vol. 1, no. 5, pp. 425-435, 2002.

[55] A. E. Frazier, C. Kiu, D. Stojanovski, N. J. Hoogenraad, and M. T. Ryan, "Mitochondrial morphology and distribution in mammalian cells," Biological Chemistry, vol. 387, no. 12, pp. 15511558, 2006.

[56] B. Westermann, "Bioenergetic role of mitochondrial fusion and fission," Biochimica et Biophysica Acta, vol. 1817, no. 10, pp. 18331838, 2012.

[57] J. C. Chang, S. J. Kou, W. T. Lin, and C. S. Liu, "Regulatory role of mitochondria in oxidative stress and atherosclerosis," World Journal of Cardiology, vol. 2, no. 6, pp. 150-159, 2010.

[58] D. C. Chan, "Mitochondria: dynamic organelles in disease, aging, and development," Cell, vol. 125, no. 7, pp. 1241-1252, 2006.

[59] K. C. Fearon, D. J. Glass, and D. C. Guttridge, "Cancer cachexia: mediators, signaling, and metabolic pathways," Cell Metabolism, vol. 16, no. 2, pp. 153-166, 2012.

[60] F. S. Lira, L. C. Carnevali, N. E. Zanchi, R. V. T. Santos, J. M. Lavoie, and M. Seelaender, "Exercise intensity modulation of hepatic lipid metabolism," Journal of Nutrition and Metabolism, vol. 2012, Article ID 809576, 8 pages, 2012.

[61] S. Lokireddy, I. W. Wijesoma, S. Teng et al., "The ubiquitin ligase mull induces mitophagy in skeletal muscle in response to muscle-wasting stimuli," Cell Metabolism, vol. 16, no. 5, pp. 613-624, 2012.

[62] M. Banasch, M. Ellrichmann, A. Tannapfel, W. E. Schmidt, and O. Goetze, "The non-invasive 13C-methionine breath test detects hepatic mitochondrial dysfunction as a marker of disease activity in non-alcoholic steatohepatitis," European Journal of Medical Research, vol. 16, no. 6, pp. 258-264, 2011.

[63] W. Dröge and H. M. Schipper, "Oxidative stress and aberrant signaling in aging and cognitive decline," Aging Cell, vol. 6, no. 3, pp. 361-370, 2007.

[64] M. E. Witte, J. J. G. Geurts, H. E. de Vries, P. van der Valk, and J. van Horssen, "Mitochondrial dysfunction: a potential link between neuroinflammation and neurodegeneration?" Mitochondrion, vol. 10, no. 5, pp. 411-418, 2010.

[65] J. A. Menendez, S. Cuf, C. Oliveras-Ferraros, L. Vellon, J. Joven, and A. Vazquez-Martin, "Gerosuppressant metformin: less is more," Aging, vol. 3, no. 4, pp. 348-362, 2011.

[66] S. I. Rattan, "Anti-ageing strategies: prevention or therapy? Showing ageing from within," EMBO Reports, vol. 6, pp. S25S29, 2005.

[67] V. B. Saprunova, M. A. Lelekova, N. G. Kolosova, and L. E. Bakeeva, "SkQ1 slows development of age-dependent destructive processes in retina and vascular layer of eyes of wistar and OXYS rats," Biochemistry, vol. 77, no. 6, pp. 648-658, 2012.

[68] T. F. Liu, C. M. Brown, M. El Gazzar et al., "Fueling the flame: bioenergy couples metabolism and inflammation," Journal of Leukocyte Biology, vol. 92, no. 3, pp. 499-507, 2012.
[69] E. Profumo, B. Buttari, L. Petrone et al., "Redox imbalance of red blood cells impacts T lymphocyte homeostasis: implication in carotid atherosclerosis," Journal of Thrombosis and Haemostasis, vol. 106, no. 6, pp. 1117-1126, 2011.

[70] C. M. Kusminski, W. L. Holland, K. Sun et al., "MitoNEETdriven alterations in adipocyte mitochondrial activity reveal a crucial adaptative process that preserves insulin sensitivity in obesity," Nature Medicine, vol. 18, no. 10, pp. 1539-1549, 2012.

[71] A. Zorzano, M. Liesa, and M. Palacín, "Role of mitochondrial dynamics proteins in the pathophysiology of obesity and type 2 diabetes," International Journal of Biochemistry and Cell Biology, vol. 41, no. 10, pp. 1846-1854, 2009.

[72] C. Aguer and M. E. Harper, "Skeletal muscle mitochondrial energetics in obesity and type 2 diabetes mellitus: endocrine aspects," Best Practice \& Research Clinical Endocrinology \& Metabolism, vol. 26, no. 6, pp. 805-819, 2012.

[73] Z. A. Ma, "The role of peroxidation of mitochondrial membrane phospholipids in pancreatic $\beta$-cell failure," Current Diabetes Reviews, vol. 8, no. 1, pp. 69-75, 2012.

[74] C. Tang, K. Koulajian, I. Schuiki et al., "Glucose-induced beta cell dysfunction in vivo in rats: link between oxidative stress and endoplasmic reticulum stress," Diabetologia, vol. 55, no. 5, pp. 1366-1379, 2012.

[75] A. Lde. Brondani, T. S. Assmann, G. C. Duarte, J. L. Gross, L. H. Canani, and D. Crispim, "The role of the uncoupling protein 1 (UCP1) on the development of obesity and type 2 diabetes mellitus," Arquivos Brasileiros de Endocrinologia e Metabologia, vol. 56, no. 4, pp. 215-225, 2012.

[76] A. Fedorenko, P. V. Lishko, and Y. Kirichok, "Mechanism of fatty-acid-dependent UCP1 uncoupling in brown fat mitochondria," Cell, vol. 151, no. 2, pp. 400-413, 2012.

[77] B. Cannon and J. Nedergaard, "Cell biology: neither brown nor white," Nature, vol. 488, no. 7411, pp. 286-287, 2012.

[78] I. Grattagliano, O. de Bari, T. C. Bernardo, P. J. Oliveira, D. Q. Wang, and P. Portincasa, "Role of mitochondria in nonalcoholic fatty liver disease-from origin to propagation," Clinical Biochemistry, vol. 45, no. 9, pp. 610-618, 2012.

[79] G. Serviddio, F. Bellanti, G. Vendemiale, and E. Altomare, "Mitochondrial dysfunction in nonalcoholic steatohepatitis," Expert Review of Gastroenterology and Hepatology, vol. 5, no. 2, pp. 233-244, 2011.

[80] N. C. Sadler, T. E. Angel, M. P. Lewis et al., "Activitybased protein profiling reveals mitochondrial oxidative enzyme impairment and restoration in diet-induced obese mice," PLoS ONE, vol. 7, no. 10, Article ID e47996, 2012.

[81] M. Carrer, N. Liu, C. E. Grueter et al., "Control of mitochondrial metabolism and systemic energy homeostasis by microRNAs 378 and 378," Proceedings of the National Academy of Sciences of the United States of America, vol. 109, no. 38, pp. 15330-15335, 2012.

[82] A. D. Karelis, M. Faraj, J. P. Bastard et al., "The metabolically healthy but obese individual presents a favorable inflammation profile," Journal of Clinical Endocrinology and Metabolism, vol. 90, no. 7, pp. 4145-4150, 2005.

[83] C. M. Kusminski and P. E. Scherer, "Mitochondrial dysfunction in white adipose tissue," Trends in Endocrinology and Metabolism, vol. 23, no. 9, pp. 435-443, 2012.

[84] A. Zorzano, M. I. Hernández-Alvarez, M. Palacín, and G. Mingrone, "Alterations in the mitochondrial regulatory pathways constituted by the nuclear co-factors PGC- $1 \alpha$ or PGC- $1 \beta$ and mitofusin 2 in skeletal muscle in type 2 diabetes," Biochimica et Biophysica Acta, vol. 1797, no. 6-7, pp. 1028-1033, 2010. 
[85] A. Zorzano, D. Sebastián, J. Segalés, and M. Palacín, “The molecular machinery of mitochondrial fusion and fission: an opportunity for drug discovery?" Current Opinion in Drug Discovery and Development, vol. 12, no. 5, pp. 597-606, 2009.

[86] X. J. Chen and R. A. Butow, "The organization and inheritance of the mitochondrial genome," Nature Reviews Genetics, vol. 6, no. 11, pp. 815-825, 2005.

[87] A. M. Distler, J. Kerner, and C. L. Hoppel, "Proteomics of mitochondrial inner and outer membranes," Proteomics, vol. 8, no. 19, pp. 4066-4082, 2008.

[88] L. Galluzzi, I. Vitale, J. M. Abrams et al., "Molecular definitions of cell death subroutines: recommendations of the nomenclature committee on cell death 2012," Cell Death and Differentiation, vol. 19, pp. 107-120, 2012.

[89] N. Joza, G. Y. Oudit, D. Brown et al., "Muscle-specific loss of apoptosis-inducing factor leads to mitochondrial dysfunction, skeletal muscle atrophy, and dilated cardiomyopathy," Molecular and Cellular Biology, vol. 25, no. 23, pp. 10261-10272, 2005.

[90] J. R. Speakman and S. E. Mitchell, "Caloric restriction," Molecular Aspects of Medicine, vol. 32, no. 3, pp. 159-221, 2011.

[91] A. Raffaello and R. Rizzuto, "Mitochondrial longevity pathways," Biochimica et Biophysica Acta, vol. 1813, no. 1, pp. 260268, 2011.

[92] N. Alkhouri, A. Gornicka, M. P. Berk et al., "Adipocyte apoptosis, a link between obesity, insulin resistance, and hepatic steatosis," The Journal of Biological Chemistry, vol. 285, no. 5, pp. 3428-3438, 2010.

[93] I. P. Salt and T. M. Palmer, "Exploiting the anti-inflammatory effects of AMP-activated protein kinase activation," Expert Opinion on Investigational Drugs, vol. 21, no. 8, pp. 1155-1167, 2012.

[94] J. G. Boyle, P. J. Logan, G. C. Jones et al., "AMP-activated protein kinase is activated in adipose tissue of individuals with type 2 diabetes treated with metformin: a randomised glycaemiacontrolled crossover study," Diabetologia, vol. 54, no. 7, pp. 1799-1809, 2011.

[95] J. E. Sullivan, K. J. Brocklehurst, A. E. Marley, F. Carey, D. Carling, and R. K. Beri, "Inhibition of lipolysis and lipogenesis in isolated rat adipocytes with AICAR, a cell-permeable activator of AMP-activated protein kinase," FEBS Letters, vol. 353, no. 1, pp. 33-36, 1994.

[96] W. Yin, J. Mu, and M. J. Birnbaum, "Role of AMP-activated protein kinase in cyclic AMP-dependent lipolysis in 3T3-L1 adipocytes," The Journal of Biological Chemistry, vol. 278, no. 44, pp. 43074-43080, 2003.

[97] M. P. Gaidhu, S. Fediuc, and R. B. Ceddia, "5-Aminoimidazole4-carboxamide-1- $\beta$-D-ribofuranoside-induced AMP-activated protein kinase phosphorylation inhibits basal and insulinstimulated glucose uptake, lipid synthesis, and fatty acid oxidation in isolated rat adipocytes," The Journal of Biological Chemistry, vol. 281, no. 36, pp. 25956-25964, 2006.

[98] M. P. Gaidhu, S. Fediuc, N. M. Anthony et al., "Prolonged AICAR-induced AMP-kinase activation promotes energy dissipation in white adipocytes: novel mechanisms integrating HSL and ATGL," Journal of Lipid Research, vol. 50, no. 4, pp. 704-715, 2009.

[99] A. T. Turer and P. E. Scherer, "Adiponectin: mechanistic insights and clinical implications," Diabetologia, vol. 55, no. 9, pp. 23192326, 2012.
[100] S. Galic, M. D. Fullerton, J. D. Schertzer et al., "Hematopoietic AMPK $\beta 1$ reduces mouse adipose tissue macrophage inflammation and insulin resistance in obesity," Journal of Clinical Investigation, vol. 121, no. 12, pp. 4903-4915, 2011.

[101] R. Vila-Bedmar, M. Lorenzo, and S. Fernández-Veledo, “Adenosine $5^{\prime}$-monophosphate-activated protein kinase-mammalian target of rapamycin cross talk regulates brown adipocyte differentiation," Endocrinology, vol. 151, no. 3, pp. 980-992, 2010.

[102] O. Horakova, D. Medrikova, E. M. van Schothorst et al., "Preservation of metabolic flexibility in skeletal muscle by a combined use of n-3 PUFA and rosiglitazone in dietary obese mice," PLoS ONE, vol. 7, no. 8, Article ID e43764, 2012.

[103] V. T. To, T. P. Hüttl, R. Lang, K. Piotrowski, and K. G. Parhofer, "Changes in body weight, glucose homeostasis, lipid profiles, and metabolic syndrome after restrictive bariatric surgery," Experimental and Clinical Endocrinology and Diabetes, vol. 120, no. 9, pp. 547-552, 2012.

[104] H. M. Heneghan, S. Nissen, and P. R. Schauer, "Gastrointestinal surgery for obesity and diabetes: weight loss and control of hyperglycemia," Current Atherosclerosis Reports, vol. 14, no. 6, pp. 579-587, 2012.

[105] A. Luke, J. O’Neill, and D. Hardie, "Metabolism of inflammation limited by AMPK and pseudo-starvation," Nature, vol. 493, pp. 346-355, 2013.

[106] C. Finelli and G. Tarantino, "Is there any consensus as to what diet of lifestyle approach is the right one for NAFLD patients?" Journal of Gastrointestinal and Liver Diseases, vol. 21, pp. 293302, 2012.

[107] J. Joven, J. Menéndez, L. Fernandez-Sender et al., "Metformin: a cheap and well-tolerated drug that provides benefits for viral infections," HIV Medicine, 2012.

[108] S. Del Barco, A. Vazquez-Martin, S. Cufí et al., "Metformin: multi-faceted protection against cancer," Oncotarget, vol. 2, no. 12, pp. 896-917, 2011.

[109] B. Viollet, B. Guigas, N. Sanz Garcia, J. Leclerc, M. Foretz, and F. Andreelli, "Cellular and molecular mechanisms of metformin: an overview," Clinical Science, vol. 122, no. 6, pp. 253-270, 2012.

[110] S. Nair, A. M. Diehl, M. Wiseman, G. H. Farr, and R. P. Perrillo, "Metformin in the treatment of non-alcoholic steatohepatitis: a pilot open label trial," Alimentary Pharmacology and Therapeutics, vol. 20, no. 1, pp. 23-28, 2004.

[111] A. Duseja, A. Das, R. K. Dhiman et al., "Metformin is effective in achieving biochemical response in patients with nonalcoholic fatty liver disease (NAFLD) not responding to lifestyle interventions," Annals of Hepatology, vol. 6, no. 4, pp. 222-226, 2007.

[112] J. W. Haukeland, Z. Konopsi, H. B. Eggesbo et al., "Metformin in patients with non-alcoholic fatty liver disease: a randomized, controlled trial," Scandinavian Journal of Gastroenterology, vol. 44, no. 7, pp. 853-860, 2009.

[113] M. Foretz, S. Hébrard, J. Leclerc et al., "Metformin inhibits hepatic gluconeogenesis in mice independently of the LKB1/AMPK pathway via a decrease in hepatic energy state," Journal of Clinical Investigation, vol. 120, no. 7, pp. 2355-2369, 2010.

[114] M. R. Owen, E. Doran, and A. P. Halestrap, "Evidence that metformin exerts its anti-diabetic effects through inhibition of complex 1 of the mitochondrial respiratory chain," Biochemical Journal, vol. 348, no. 3, pp. 607-614, 2000.

[115] B. H. Ahn, H. S. Kim, S. Song et al., "A role for the mitochondrial deacetylase Sirt3 in regulating energy homeostasis," Proceedings of the National Academy of Sciences of the United States of America, vol. 105, no. 38, pp. 14447-14452, 2008. 
[116] X. Kong, R. Wang, Y. Xue et al., "Sirtuin 3, a new target of PGC- $1 \alpha$, plays an important role in the suppression of ROS and mitochondrial biogenesis," PLoS ONE, vol. 5, no. 7, Article ID el1707, 2010.

[117] M. Buler, S. M. Aatsinki, V. Izzi, and J. Hakkola, "Metformin reduces hepatic expression of SIRT3, the mitochondrial deacetylase controlling energy metabolism," PLoS ONE, vol. 7, no. 11, Article ID e49863, 2012.

[118] P. W. Caton, N. K. Nayuni, J. Kieswich, N. Q. Khan, M. M. Yaqoob, and R. Corder, "Metformin suppresses hepatic gluconeogenesis through induction of SIRT1 and GCN5," Journal of Endocrinology, vol. 205, no. 1, pp. 97-106, 2010.

[119] B. Corominas-Faja, R. Quirantes-Piné, C. Oliveras-Ferraros et al., "Metabolomic fingerprint reveals that metformin impairs one-carbon metabolism in a manner similar to the antifolate class of chemotherapy drugs," Aging, vol. 4, no. 7, pp. 480-498, 2012.

[120] R. Beltrán-Debón, A. Rull, F. Rodríguez-Sanabria et al., "Continuous administration of polyphenols from aqueous rooibos (Aspalathus linearis) extract ameliorates dietaryinduced metabolic disturbances in hyperlipidemic mice," Phytomedicine, vol. 18, no. 5, pp. 414-424, 2011.

[121] J. Joven, A. Rull, E. Rodríguez-Gallego et al., "Multifunctional targets of dietary polyphenols in disease: a case for the chemokine network and energy metabolism," Food and Chemical Toxicology, vol. 51, pp. 267-279, 2013.

[122] J. Joven, E. Espinel, A. Rull et al., "Plant-derived polyphenols regulate expression of miRNA paralogs miR-103/107 and miR122 and prevent diet-induced fatty liver disease in hyperlipidemic mice," Biochimica et Biophysica Acta, no. 7, pp. 894-899, 1820.

[123] A. Segura-Carretero, M. A. Puertas-Mejía, S. CortaceroRamírez et al., "Selective extraction, separation, and identification of anthocyanins from Hibiscus sabdariffa L. using solid phase extraction-capillary electrophoresis-mass spectrometry (time-of-flight/ion trap)," Electrophoresis, vol. 29, no. 13, pp. 2852-2861, 2008.

[124] S. C. Johnson, P. S. Rabinovitch, and M. Kaeberlein, "mTOR is a key modulator of ageing and age-related disease," Nature, vol. 493, pp. 338-345, 2013.

[125] M. Herranz-López, S. Fernández-Arroyo, A. Pérez-Sanchez et al., "Synergism of plant-derived polyphenols in adipogenesis: perspectives and implications," Phytomedicine, vol. 19, no. 3-4, pp. 253-261, 2012.

[126] S. Virtue and A. Vidal-Puig, "It's not how fat you are, it's what you do with it that counts," PLoS Biology, vol. 6, no. 9, article e237, 2008.

[127] R. Singh and A. M. Cuervo, "Lipophagy: connecting autophagy and lipid metabolism," International Journal of Cell Biology, vol. 2012, Article ID 282041, 12 pages, 2012.

[128] H. J. Jansen, P. van Essen, T. Koenen, L. A. Joosten, M. G. Netea, and C. J. Tack, "Autophagy activity is up-regulated in adipose tissue of obese individuals and modulates proinflammatory cytokine expression," Endocrinology, vol. 153, no. 12, pp. 58665874, 2012. 


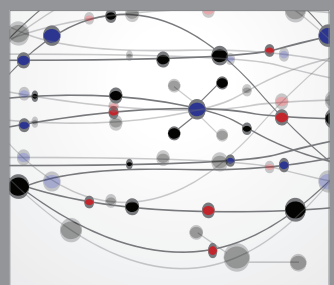

The Scientific World Journal
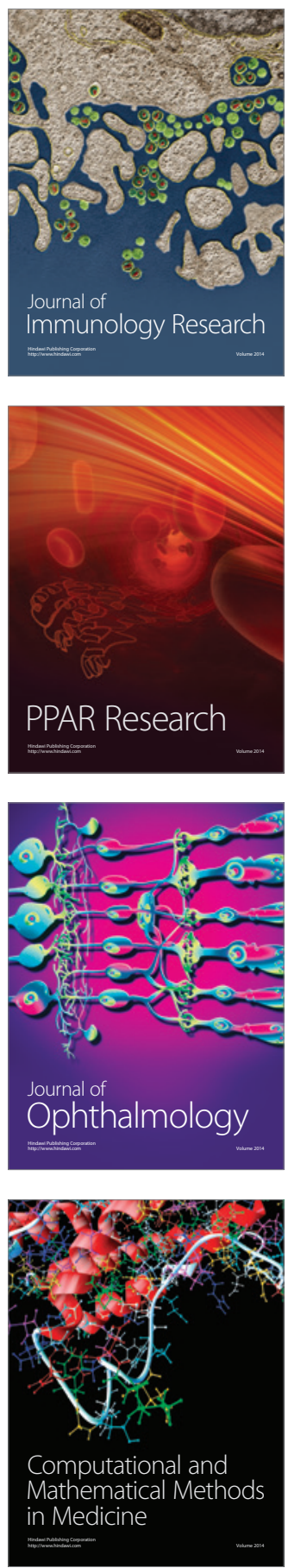

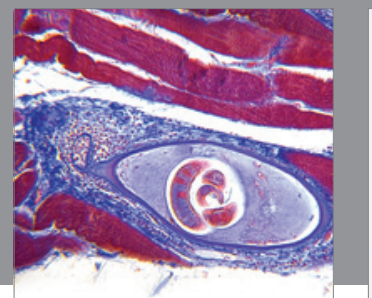

Gastroenterology

Research and Practice


\section{Hindawi}

Submit your manuscripts at

http://www.hindawi.com
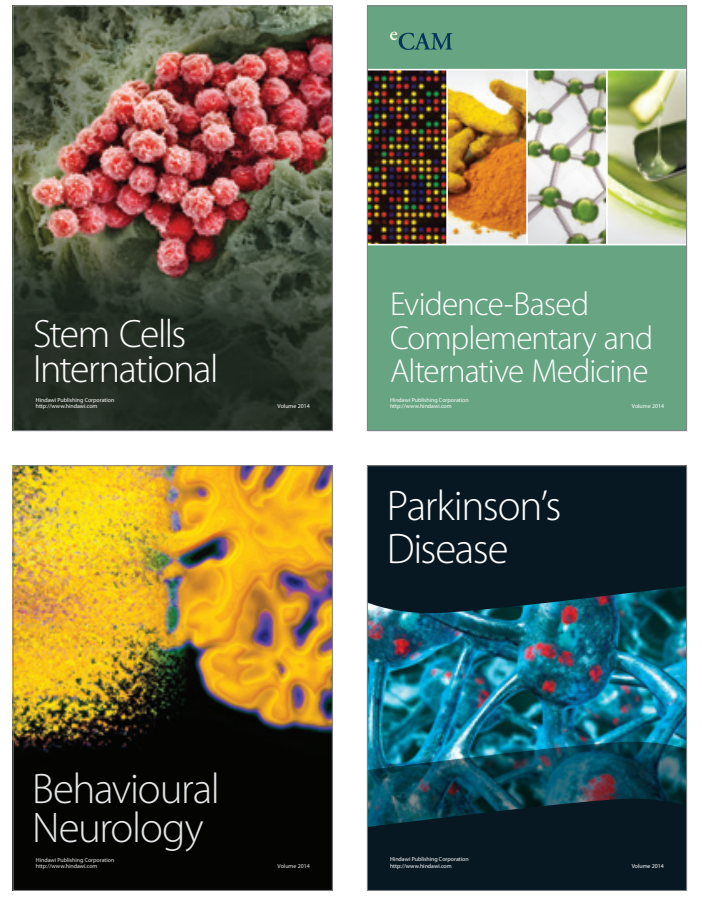
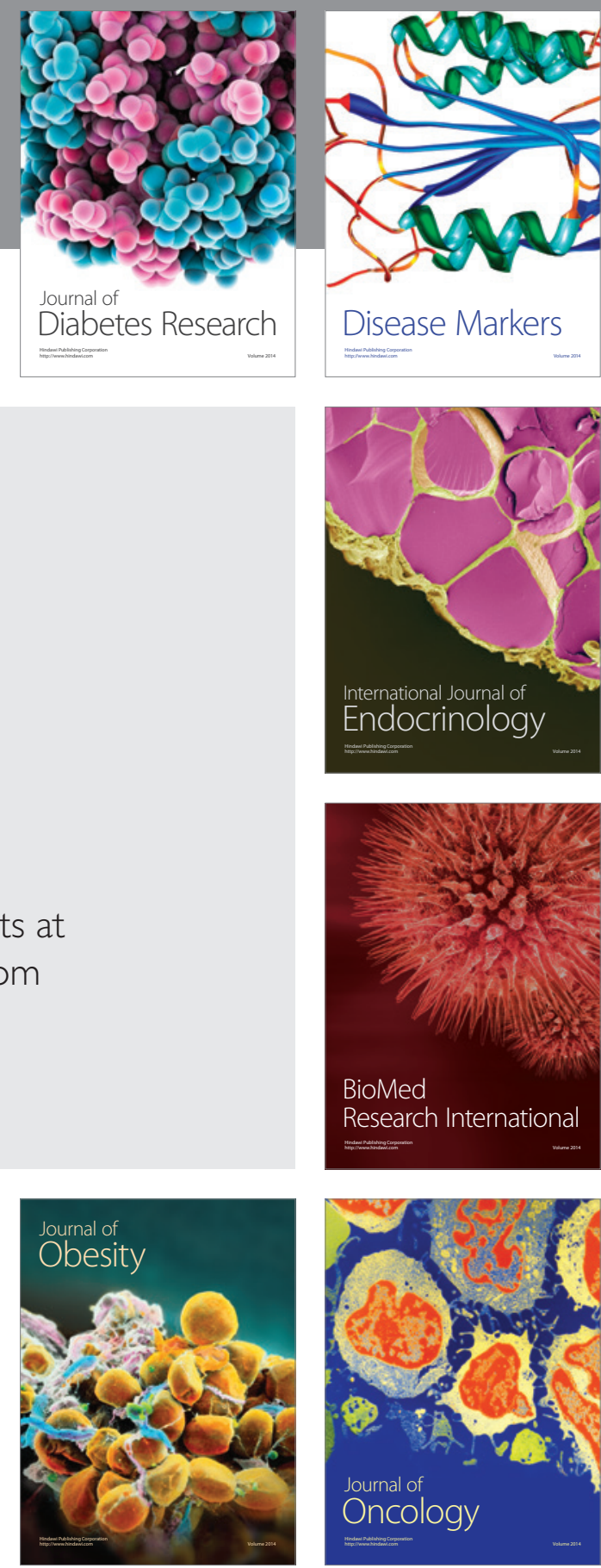

Disease Markers
